\title{
Condições de saúde bucal de idosos da instituição de longa permanência Lar Samaritano no município de São Gonçalo-RJ
}

\author{
Oral health conditions of the elderly at the Lar Samaritano \\ long-term-care facility in São Gonçalo-RJ
}

\author{
Ingrid Petra Chaves Sá ${ }^{1}$ \\ Levi Ribeiro de Almeida Júnior ${ }^{1}$ \\ Marcos Paulo Fonseca Corvino ${ }^{1}$ \\ Selma Petra Chaves Sá ${ }^{2}$
}

${ }^{1}$ Faculdade de Odontologia, Universidade Federal Fluminense. Rua São Paulo 28 Campus do

Valonguinho, Centro. 24020-150 Niteroi RJ. ingridpetra@hotmail.com ${ }^{2}$ Departamento de Fundamento de Enfermagem, Escola de Enfermagem, Universidade Federal Fluminense.
Abstract With the increase in life expectancy in Brazil, there are increasing numbers of elderly. There is a marked increase in demand for LongTerm-Care Facilities for the elderly in Brazil, but there is little epidemiological data on oral health in such institutions. The objective is to evaluate the oral health of elderly residents in a LongTerm-Care Facility in São Gonçalo city, Rio de Janeiro, examining dental conditions, the use of and need for prostheses and dental treatment. Quantitative research was conducted with functionally dependent and/or independent male and female subjects over 59 years of age, involving data gathering, consulting records, interviews and clinical examinations. DMFT 30.37, predominantly missing teeth and the female population with fewer decayed teeth. Only 2 had excellent hygiene, 25 were good, 17 satisfactory and $14 \mathrm{bad}$. Tooth mobility was found in 6 elderly though 52 had no mobility, while 32 wore dentures and 26 did not, with women using them more frequently. 49 lacked treatment, mainly women as they were greater in number. The conclusion reached is that Long-Term-Care Facilities need inspection by public and dental authorities, with priority given to dental professionals, in addition to fostering academic activities for undergraduate and postgraduate students.

Key words Dentistry, Elderly, Oral health
Resumo Com aumento da expectativa de vida brasileira, cresce o número de idosos. Observa-se maior demanda deles por Instituições de Longa Permanência (ILP) no Brasil, contudo são escassos dados epidemiológicos sobre saúde bucal nestas. O objetivo é avaliar as condições de saúde bucal de idosos residentes numa ILP, no município São Gonçalo (RJ), levantando a condição dentária, o uso e a necessidade de prótese e de tratamento dentário. Pesquisa quantitativa com indivíduos dos sexos masculino e feminino, funcionalmente dependentes e/ou independentes. Coleta de dados: consulta ao prontuário, entrevista e exame clínico bucal. A faixa etária foi a partir de 59 anos. CPO-D 30,37, predominando o componente perdido, tendo o grupo feminino menor número de dentes cariado. Apenas 2 apresentavam higienização ótima, 25 boa, 17 regular e 14 péssima. Mobilidade dental em 6 idosos e 52 sem mobilidade. Trinta e dois usavam prótese dentária e 26 não, mulheres com maior frequência de uso. Quarenta e nove careciam de tratamento, sendo as mulheres quem mais necessitava por estarem em número maior. Concluiu-se que as ILP necessitam de fiscalização dos órgãos públicos e odontológicos, priorizando e reivindicando profissionais de odontologia, além de estimular atividades acadêmicas para alunos da graduação e pós-graduação.

Palavras-chave Odontologia, Idoso, Saúde bucal 


\section{Introdução}

Segundo os resultados da Pesquisa Nacional por Amostra de Domicílios ${ }^{1}$, a expectativa de vida do brasileiro elevou-se para 71,7 anos, e a população de 60 anos ou mais, passou a representar 9,7\% do total. Ressalta-se ainda que segundo o Censo de $2000^{2}$, houve um aumento considerável dos mais velhos, tendo a população de 80 anos e mais registrado acréscimo de $12,6 \%$.

A família tem encontrado crescentes dificuldades para desempenhar determinadas atividades como cuidar de seus idosos em seu próprio domicílio. Com isso, a expressão "intimidade à distância” (diferentes gerações ou mesmo pessoas de uma mesma família ocupam residências separadas) torna-se cada vez mais conhecida na gerontologia, onde o idoso passa por um grande desafio, mudando da sua própria moradia para uma Instituição de Longa Permanência (ILP), necessitando de abordagem especial, de acompanhamento e apoio.

No passado e ainda hoje, há um grande número de ILP funcionando com uma equipe mínima, constituída por uma assistente social e auxiliar de enfermagem ou cuidador de idoso, além de pessoal de apoio. Com isso, observa-se que, a maioria das instituições não possui um cirurgião-dentista para atendimento e avaliação bucal dos idosos residentes.

Assim, o objetivo deste estudo consiste em avaliar as condições de saúde bucal de idosos residentes em uma Instituição de Longa Permanência, no município de São Gonçalo, no Estado do Rio de Janeiro (RJ), onde se levantou a condição dentária, a condição da higiene bucal, a condição periodontal, como mobilidade, uso, tipo e necessidade de prótese, a higienização das próteses, a adequação destas e a necessidade de tratamento dentário.

Instituição de Longa Permanência para Idosos corresponde às instituições governamentais ou não-governamentais, de caráter residencial, destinadas a domicílio coletivo de pessoas com idade igual ou superior a 60 anos, com ou sem suporte familiar, em condições de liberdade e dignidade e cidadania, conforme regulamento Técnico para o Funcionamento das Instituições de Longa Permanência para Idosos - RDC n 283, de 26 de setembro de $2005^{3}$.

Para a Sociedade Brasileira de Geriatria e Gerontologia ${ }^{4}$ são estabelecimentos para atendimento integral institucional, cujo público alvo é constituído por pessoas de 60 anos e mais, dependentes ou independentes, que não dispõem de condi- ções para permanecer com a família ou em seu domicílio. Estas instituições, conhecidas por denominações diversas - abrigo, asilo, lar, casa de repouso, clínica geriátrica e ancianato -, devem proporcionar serviços na área social, médica, de psicologia, de enfermagem, de fisioterapia, de terapia ocupacional, de odontologia, e em outras, conforme as necessidades desse segmento etário.

Nas Políticas Públicas direcionadas aos idosos, recomendam a permanência e o cuidado deles como atividade de sua própria família, exceto nos casos em que ambos careçam de condições mínimas de sobrevivência.

Os muitos fatores que levam a institucionalização do idoso, como morar só, ter suporte social precário e baixa renda, associados à viuvez, à aposentadoria, à menor oportunidade de empregos formais e estáveis e ao aumento dos gastos com a própria saúde, tornaram-se mais frequentes nos últimos anos.

A internação do idoso em uma instituição de longa permanência pode se apresentar como única saída para a família, frente à não disponibilidade dos suportes familiar, financeiro e psicológico que o mesmo necessita. Nestas instituições, o indivíduo vive na forma de internato por tempo determinado ou não, mediante pagamento ou não.

A precariedade da situação de saúde bucal dos idosos institucionalizados é notória, entretanto dados epidemiológicos ainda são escassos por ser um assunto pouco explorado, mesmo em outros países do mundo. Pode-se mencionar um estudo epidemiológico realizado em uma instituição de longa permanência na cidade de Helsinki, Finlândia, onde observou-se que $42 \%$ dos idosos examinados eram desdentados. Dos dentados, 37\% necessitavam de restaurações, 51\% de terapia periodontal e $42 \%$ de extrações ${ }^{5}$.

Em idosos residentes em instituições na cidade de Berlim (Alemanha), apenas 37,6\% das próteses apresentavam retenção e estabilidade adequadas. Em $80 \%$ dos pacientes o tratamento odontológico era necessário ${ }^{6}$.

No Brasil, um estudo epidemiológico em instituições de longa permanência no município de Araçatuba, SP, mostrou que 69\% dos idosos eram desdentados totais, $48 \%$ eram portadores de prótese total e $52 \%$ não usavam prótese ${ }^{7}$. No município de Araraquara, São Paulo, 72\% dos idosos institucionalizados eram desdentados. Cerca de $90 \%$ dos dentes já estavam perdidos e $61 \%$ dos examinados necessitavam de prótese dentária ${ }^{8}$.

Outro estudo com idosos institucionalizados em Curitiba, 56 eram desdentados totais e 51 
desdentados parciais. Dos desdentados totais, 44 (79\%) faziam uso de pelo menos uma prótese total e $12(21 \%)$ não usavam nenhum tipo de prótese ${ }^{9}$.

Assim, a presente pesquisa se justifica pelo crescente número de ILP, pela falta de cirurgiões dentistas nestas instituições e pelos levantamentos no país e no exterior, os quais demonstram os diversos problemas e intercorrências relacionados à saúde bucal dos idosos nestas instituições.

A relevância deste estudo decorre da importância social da pesquisa na avaliação das reais condições bucais dos idosos residentes em instituição de longa permanência. Além disso, a contribuição se dá a partir do momento em que, com os dados, pode-se identificar os problemas bucais mais recorrentes na ILP, direcionar as intervenções preventivas e terapêuticas, prescrever a higiene bucal diária e aplicar fluoretos tópicos e soluções bucais para evitar a recorrência e a progressão das doenças bucais, melhorando, assim, a qualidade de vida do idoso residente em Instituições e também contribuir para pesquisas sobre o tema na odontogeriatria, já que há uma grande carência de trabalhos que abordem tal assunto.

\section{Material e métodos}

Trata-se de um estudo com abordagem quantitativa, com sujeitos a partir de 59 anos de idade, residentes na Instituição de Longa Permanência conhecida como Lar Samaritano no Município de São Gonçalo, Rio de Janeiro. Apesar de ser uma instituição de longa permanência para idosos, encontravam-se alguns indivíduos com idade inferior a 60 anos, que foram incluídos na pesquisa por serem residentes na instituição.

A pesquisa foi realizada com 58 indivíduos tanto do sexo masculino como do feminino, funcionalmente dependentes e/ou independentes residentes na instituição acima mencionada. Para garantir o anonimato dos sujeitos, eles foram identificados por números e pela letra $\mathrm{M}$ quando do sexo masculino e $\mathrm{F}$ quando do feminino.

A coleta de dados se deu em três momentos: consulta ao prontuário, entrevista e exame clínico bucal dos idosos no período de janeiro a junho de 2009.

Dos prontuários foram obtidos dados como idade, sexo, data de nascimento, estado civil e tempo na instituição.

Foi elaborado um formulário para entrevista com perguntas fechadas, abordando doenças sistêmicas, presença de queixas relacionadas à cavidade bucal, à higiene bucal, aos cuidados com as próteses e às necessidades de tratamento odontológico. Caso o idoso apresentasse dificuldades para responder às questões da entrevista, esta era realizada com o cuidador da instituição.

Em seguida, foi realizado exame clínico bucal para avaliação das condições de saúde bucal, como: condições dentárias através do CPO-D (número médio de dentes permanentes cariados, perdidos e obturados, em determinado espaço geográfico); condições da higiene bucal; condição periodontal, como mobilidade, presença de dentes hígidos e de dentes na cavidade bucal, assim como a de restos radiculares; uso, tipo e necessidade, higienização e adequação das próteses dentárias; e, a necessidade de tratamento dentário.

Para avaliação da condição periodontal utilizou-se apenas o critério da presença de mobilidade ou não.

Em relação ao uso de próteses dentárias verificou-se a adaptação, o tipo de prótese, se é total, parcial removível à grampo ou fixa, sua funcionalidade na cavidade bucal, o estado de higienização, a remoção durante a noite e a necessidade de usar algum outro tipo que lhe pudesse conferir melhor adaptação.

Os achados foram digitados e apresentados através de quadros, tabelas e gráficos no Programa Office Word 2003 e analisados a partir de autores que abordaram o assunto.

Para os idosos com distúrbios cognitivos ou com alguma limitação que dificultasse o entendimento da pesquisa, ou em assinar o termo de consentimento livre e esclarecido, os seus responsáveis foram contactados e esclarecidos sobre a pesquisa para autorização. Com relação aos idosos com distúrbios cognitivos e limitações em compreender a pesquisa e que não tinham um responsável familiar, foi solicitado a assinatura do responsável técnico da instituição para a coleta de dados.

O protocolo de pesquisa foi encaminhado para apreciação do Comitê de Ética e aprovado.

\section{Resultados e discussão}

A coleta dos dados ocorreu com 58 idosos, sendo que desses, $36(62,06 \%)$ eram do sexo feminino e $22(37,93 \%)$ do masculino, embora um número maior de mulheres residisse na instituição, conforme Tabela 1.

Segundo dados do IBGE, citados por Camarano ${ }^{10}$, o número de mulheres idosas é maior do que o de homens, visto que elas possuem uma 
perspectiva de vida maior. Segundo Sá ${ }^{11}$, alguns autores chegam a chamar este fenômeno, de "feminilização da velhice”.

De acordo com a American Geriatrics Society $^{12}$ quase a metade das pessoas que vivem nessas instituições tem 85 anos ou mais, a maioria são mulheres (72\%) e com um grupo pequeno de familiares e amigos para dar suporte.

Em relação à faixa etária dos idosos, teve-se como corte a partir de 59 anos, sendo 10 idosos entre 59-69 (17,24\%), 21 (36,20\%) entre 70-79, igual número para os idosos na faixa de $80-89$ anos e somente $6(10,34 \%)$ com idade a partir de 90 anos.

Quanto ao estado civil, somente um idoso era casado, enquanto 22 (vinte e dois) eram solteiros, 5 (cinco) divorciados e 28 (vinte e oito) viúvos. A maioria era de solteiros ou viúvos, podendo, assim, justificar a institucionalização.

$\mathrm{Na}$ Tabela 2, apresenta os dados levantados por meio do CPO-D dos idosos institucionalizados. O CPO-D teve como um total de 30,37, sendo considerado um alto grau de severidade, havendo predomínio do componente perdido que representou 1.707 dentes perdidos, dos quais 627 eram do sexo masculino e 1.080 do sexo feminino, tendo este último grupo o menor número de cariados (dez) em comparação aos homens com dezessete, até porque apresentam menor quantidade de dentes presentes, sendo a grande maioria desdentados.

A Organização Mundial de Saúde (OMS) havia estabelecido como meta para o ano 2000 que $50 \%$ das pessoas na faixa etária de 65-74 anos apresentassem pelo menos 20 dentes em condições funcionais ${ }^{13}$. Já no último levantamento epidemiológico nacional das condições de saúde bucal da população brasileira, que se deu em 2002 e 2003, no qual foram avaliados mais de cinco mil idosos, tendo sido estabelecido como SB Brasil 2003 (Condições de Saúde Bucal da população brasileira) revelou que o índice CPO-D (den- tes cariados, perdidos e obturados) médio na população idosa com idade entre 65-74 anos foi de 27,79 tendo o componente dente perdido elevado para $25,83^{13}$. Os dados encontrados em outro estudo ${ }^{14}$ e na instituição Lar Samaritano, em São Gonçalo (RJ), de índice CPO-D 30,37, com o componente $\mathrm{P}$ igual a 1.707, confirmaram essa situação.

Conforme menciona Trelha et al. ${ }^{14}$, muitos outros autores encontraram valores maiores para o CPO-D por terem analisados população que incluíam idosos com mais de 74 anos de idade, assim como esta pesquisa.

Outra diferença, em relação ao índice CPO$D$, se destaca na verificação dos dados deste estudo, cuja amostra é composta por idosos institucionalizados enquanto que no levantamento nacional foram examinados idosos em seus domicílios. Segundo Trelha et al. ${ }^{14}$, os idosos que moram em seu domicílio com suas famílias ou cuidadores e que são funcionalmente independentes apresentam menos interferência das comorbidades comumente encontrados naqueles institucionalizados que podem ou não apresentar alto grau de dependência para realização das AVD, não sendo diferente com os cuidados necessários a sua saúde bucal.

Tabela 2. Condição dentária de 58 idosos da Instituição de Longa Permanência Lar Samaritano - SG/RJ, 2009.

\begin{tabular}{lrrr}
\hline $\begin{array}{c}\text { CPO-D e } \\
\text { componentes }\end{array}$ & $\begin{array}{c}\text { Feminino } \\
\mathbf{n}^{\mathbf{0}}\end{array}$ & $\begin{array}{c}\text { Masculino } \\
\mathbf{n}^{\mathbf{0}}\end{array}$ & $\begin{array}{c}\text { Total } \\
\mathbf{n}^{\mathbf{0}}\end{array}$ \\
\hline Cariados & 10 & 17 & 27 \\
Perdidos & 1.080 & 627 & 1.707 \\
Obturados & 19 & 09 & 28 \\
Dentes & 72 & 74 & 146 \\
CPO-D & 30,80 & 29,68 & 30,37 \\
\hline
\end{tabular}

Tabela 1. Sexo e Faixa etária de 58 idosos da Instituição de Longa Permanência Lar Samaritano - SG/RJ, 2009.

\begin{tabular}{|c|c|c|c|c|c|c|}
\hline \multirow[b]{2}{*}{ Faixa etária } & \multicolumn{2}{|c|}{ Feminino } & \multicolumn{2}{|c|}{ Masculino } & \multicolumn{2}{|c|}{ Total } \\
\hline & $\mathbf{N}$ & $\%$ & $\mathbf{N}$ & $\%$ & $\mathrm{~N}$ & $\%$ \\
\hline 59-69 anos & 05 & $13,89 \%$ & 05 & $22,73 \%$ & 10 & $17,24 \%$ \\
\hline 70-79 anos & 13 & $36,11 \%$ & 08 & $36,36 \%$ & 21 & $36,21 \%$ \\
\hline 80-89 anos & 12 & $33,33 \%$ & 09 & $40,91 \%$ & 21 & $36,21 \%$ \\
\hline A partir de 90 anos & 06 & $16,67 \%$ & 0 & $0 \%$ & 06 & $10,34 \%$ \\
\hline Total & 36 & $100,00 \%$ & 22 & $100,00 \%$ & 58 & $100,00 \%$ \\
\hline
\end{tabular}


Em pesquisa realizada em Londrina, Paraná, o índice de dentes cariados, perdidos e obturados (CPO-D) foi de 27,9, com maior participação dos dentes perdidos (85,9\%). O edentulismo foi detectado em $43,1 \%$ dos idosos, e a presença de 20 dentes naturais ou mais em 8,8\% das mulheres e $28 \%$ dos homens. A necessidade de prótese foi de $45,7 \%$ na arcada inferior e de $19,1 \%$ na superior $^{14}$.

Em outra pesquisa, realizada em Goiânia (GO), foi obtido o CPO-D médio de 30,17, havendo predomínio do componente extraído. Quase metade, 49,48\%, usava prótese e 80,28\% necessitava de alguma prótese ${ }^{15}$. Com esses dados, verifica-se que o edentulismo é ainda um dos grandes problemas de saúde pública em diferentes localidades.

Em relação à higiene bucal, dos 58 sujeitos, 2 idosos apresentavam ótima higienização, 25 boa, 17 regular e 14 péssima.

A avaliação da mobilidade dental estava presente em apenas 6 idosos, enquanto 52 não a apresentavam, até pelo fato dos idosos apresentarem muitos dentes perdidos na cavidade bucal.

Quanto às doenças periodontais, estas podem se manifestar desde a forma de uma gengivite até a de periodontites severas com consequente mobilidade e perda do elemento dentário, podendo levar ao uso de próteses. Apesar de comum em idosos, a doença periodontal, levando à mobilidade, não é consequência da idade.

De acordo com a Tabela 3, sobre o uso de prótese dentária, $32(55,17 \%)$ faziam uso desta, enquanto $26(44,82 \%)$ não, tendo sido verificado que as mulheres a usavam com mais frequência que os homens. Para a arcada superior, 31 $(53,44 \%)$ idosos usavam Prótese Total, apenas 1 $(1,72 \%)$ Prótese Parcial Removível e 26 não usavam nenhum tipo. Já para arcada inferior, 16 $(27,58 \%)$ idosos usavam Prótese Total, 2 (3,44\%) Prótese Parcial Removível. Houve um número inexpressivo de uso de outros tipos de próteses que não a total.

A grande maioria dos pacientes geriátricos apresenta, em nível de saúde bucal, um quadro clínico comum, caracterizado por edentulismo, presença de próteses (totais, parciais, fixas e/ou removíveis), ausência de vários elementos dentários, doença periodontal e neoplasias malignas.

Slade et al. ${ }^{16}$ também compararam as condições de saúde bucal em idosos institucionalizados e não institucionalizados em Ontário, Canadá, concluindo que primeiros apresentam duas vezes mais chances de serem edentados.

Em relação ao uso de próteses dentárias, as totais são predominantes, sendo mais utilizadas na arcada superior do que na inferior, por ser difícil de alcançar a estabilidade e retenção nesta última, devido à reabsorção óssea pelas inúmeras extrações e pela idade avançada, comprometendo a adaptação da prótese na mucosa.

Dos 32 idosos que faziam uso de prótese dentária, 18 estavam com a prótese adequada e 14 não.

Observando a higienização das próteses dentárias, $22(37,93 \%)$ apresentavam-se boas. Foi

Tabela 3. Uso e Tipo de Prótese Dentária na ILP Lar Samaritano - SG/RJ, 2009.

\begin{tabular}{|c|c|c|c|}
\hline \multirow{2}{*}{$\begin{array}{l}\text { Uso de prótese e tipo } \\
\text { de prótese dentária }\end{array}$} & Mulheres & Homens & Total \\
\hline & $\mathrm{N}$ & $\mathrm{N}$ & $\mathrm{N}$ \\
\hline Usa & 18 & 14 & 32 \\
\hline Não usa & 18 & 08 & 26 \\
\hline Total & 36 & 22 & 58 \\
\hline
\end{tabular}

\begin{tabular}{|c|c|c|c|c|c|c|c|c|}
\hline & \multicolumn{8}{|c|}{ Arcada dentária } \\
\hline & \multicolumn{4}{|c|}{ Superior } & \multicolumn{4}{|c|}{ Inferior } \\
\hline & \multicolumn{2}{|c|}{ Mulher } & \multicolumn{2}{|c|}{ Homem } & \multicolumn{2}{|c|}{ Mulher } & \multicolumn{2}{|c|}{ Homem } \\
\hline & $\mathbf{N}$ & $\%$ & $\mathbf{N}$ & $\%$ & $\mathbf{N}$ & $\%$ & $\mathbf{N}$ & $\%$ \\
\hline PT & 18 & 100,00 & 13 & 92,86 & 09 & 50,00 & 07 & 50,00 \\
\hline PPR & 0 & 0 & 01 & 7,14 & 0 & 0 & 02 & 14,29 \\
\hline $\mathrm{PF}$ & 0 & 0 & 0 & 0 & 0 & 0 & 0 & 0 \\
\hline Não usa & - & - & - & - & 09 & 50,00 & 05 & 35,71 \\
\hline Total & 18 & 100,00 & 14 & 100,00 & 18 & 100,00 & 14 & 100,00 \\
\hline
\end{tabular}


relatado pelos cuidadores que não é comum fazer a higienização diariamente porque são muitos idosos para poucos cuidadores. Conclui-se, então, que houve alguma interferência, provavelmente por ter sido feito a comunicação prévia acerca da pesquisa e as datas do exame bucal. A maioria, 20 idosos, não as removiam à noite.

Assim, conforme apresentado na Tabela 4, em relação à necessidade de tratamento odontológico, dos 58 idosos residentes na instituição, 49 (84,48\%) careciam de algum tipo de tratamento, sendo as mulheres (31) quem mais necessitava desta assistência, até por serem em número maior.

Os tratamentos necessários na instituição, mais evidentes, foram a exodontia e a confecção de próteses dentárias, por conta do edentulismo. Este provoca a perda de apetite pelo fato dos idosos não conseguirem se alimentar corretamente e de acordo com as suas necessidades. Deste modo, recorrem à substituição dos dentes pelas próteses dentárias.

Em relação à exodontia, alguns dentes precisavam ser extraídos em decorrência da mobilidade, e/ou de estarem comprometidos por cáries extensas e profundas, e/ou por restaurações mal adaptadas, devido à falta de consultas odontológicas.

Observou-se, também, a necessidade da confecção de prótese dentária devido ao grande número de desdentados, os quais não faziam uso, enquanto algumas próteses dos idosos não estavam apropriadas para que o aparelho fonéticomastigatório estivesse reabilitado.

\section{Considerações finais}

A proposta desta pesquisa foi analisar as condições bucais dos idosos residentes na Instituição de Longa Permanência Lar Samaritano, visando conhecer as necessidades de tratamento odonto-

Tabela 4. Necessidade de Tratamento odontológico de acordo com a faixa etária dos idosos da ILP Lar Samaritano - SG/RJ, 2009.

\begin{tabular}{lrrrrr}
\hline & \multicolumn{2}{c}{ Homem } & & \multicolumn{2}{c}{ Mulher } \\
\cline { 2 - 3 } \cline { 6 - 7 } \multicolumn{1}{c}{ Faixa etária } & Sim & Não & & Sim & Não \\
\hline $59-69$ anos & 04 & 01 & & 05 & 0 \\
$70-79$ anos & 06 & 02 & & 12 & 01 \\
$80-89$ anos & 08 & 01 & & 9 & 03 \\
A partir de 90 anos & 0 & 0 & & 5 & 01 \\
Total & 18 & 04 & & 31 & 05 \\
\hline
\end{tabular}

lógico, de modo a poder propor uma melhoria tanto na qualidade de vida como na dos padrões de saúde bucal.

Assim, a situação de saúde bucal dos idosos pesquisados é precária, especialmente devido à alta prevalência de edentulismo, registrando um número elevado de dentes perdidos, o que constitui um problema grave de saúde bucal que remete a uma reflexão sobre a prática odontológica mutiladora e à iniquidade no acesso aos serviços odontológicos aos quais os idosos foram submetidos no passado.

A grande necessidade de prótese evidencia a falta de ações reabilitadoras ofertadas pelo serviço público, ratificando a ausência de políticas públicas de saúde bucal voltadas para este grupo que se encontra institucionalizado. Além disso, a própria institucionalização pode comprometer a socialização e, acresce a isto, a precária condição bucal dos sujeitos residentes nestes locais, tendo em vista que a cavidade bucal, e seus componentes, são essenciais para a socialização e, consequentemente, favorecendo a melhoria da qualidade de vida das pessoas.

Pode-se enfatizar que as ILP necessitam de fiscalização dos órgãos públicos e odontológicos, inclusive, priorizando e reivindicando profissionais de odontologia em suas equipes de fiscalização. Os currículos dos cursos de odontologia devem estimular as atividades acadêmicas nestas instituições, pois são consideradas locais férteis para os alunos da graduação e os da pós-graduação.

Ressalta-se, por fim, que a Odontogeriatria precisa ter bases para uma visão geral do idoso e do envelhecer, sendo importante estabelecer programas de saúde bucal que atendam às necessidades específicas da população idosa, tanto em nível preventivo quanto curativo, e como prioridades frente às necessidades, encontradas nesta pesquisa, para que as condições de saúde bucal necessárias para viver com qualidade nesta etapa da vida sejam mantidas, assim como a reabilitação do aparelho fonético-mastigatório, também relevantes. 


\section{Colaboradores}

IPC Sá e LR Almeida Júnior participaram da coleta e da análise de dados; MPF Corvino participou da análise de dados e compartilhamento da revisão geral; e, SPC Sá da análise de dados.

\section{Referências}

1. Instituto Brasileiro de Geografia e Estatística (IBGE). Pesquisa Nacional por Amostra de domicílios. Síntese de indicadores 2005. [site na Internet]. [acessado 2012 abr 7]. Disponível em: http://www.ibge.gov.br /home/estatistica/populacao/trabalhoerendimento/ pnad2005/default.shtm

2. Instituto Brasileiro de Geografia e Estatística (IBGE). Censo 2000. [site na Internet]. [acessado $2012 \mathrm{abr}$ 7]. Disponível em: http://www.ibge.gov.br/censo/

3. Freitas EV, Py L, Cançado FAX, Doll J, Gorzoni ML. Tratado de Geriatria e Gerontologia. Rio de Janeiro: Guanabara Koogan, 2002.

4. Sociedade Brasileira de Geriatria e Gerontologia (SBGG). [site na Internet]. [acesso 2009 ago 03]. Disponível em: http//www.sbggrj.org.br/

5. Peltola P, Vehkalahti MM, Wuolijoki-Saaristo K. Oral health and treatment needs of the long-term hospitalized elderly. Gerodontology 2004; 21(2):93-99.

6. Knabe C, Kram P. Dental care for institutionalized geriatric patients in Germany. J Oral Rehabil 1997; 21(12):909-912.

7. Saliba CA, Saliba, NA, Marcelino G, Moimaz SA. Saúde bucal dos idosos: uma realidade ignorada. Rev Assoc Paul Cir Dent 1999; 53(4):279-282.

8. Silva SRC, Valsecki Junior A. Avaliação das condições de saúde bucal dos idosos em um município brasileiro. Rev Panam Salud Publica 2000; 8(4):268271.

9. Ditterich RG, Rodrigues CK, Hebling E. Atenção bucal ao idoso institucionalizado: uma lacuna na odontologia. Medcenter Odontologia [periódico on line]. 2004. [acessado 2010 abr 7]. Disponível em: http:// www.odontologia.com.br/artigos.asp?id $=464$

10. Camarano AA. Envelhecimento da população brasileira: uma contribuição demográfica. In: Freitas EV, Py L, Cançado FAX, Doll J, Gorzoni ML. Tratado de Geriatria e Gerontologia. Rio de Janeiro: Guanabara; 2002.
11. Sá SPC. Idoso: representação social da velhice e as implicações no cuidado de si [tese]. Rio de Janeiro (RJ): Universidade Federal do Rio de Janeiro; 2004.

12. American Geriatrics Society (AGS). Foundation for Health in Aging. Aging in the Know. Nursing Home Care. [site na Internet]. [acesso 2009 ago 03]. Disponível em: URL: http//www.nlm.nih.go

13. Gaião L, Almeida M. Perfil Epidemiológico da cárie dentária, doença periodontal, uso e necessidade de prótese em idosos residentes em uma instituição na cidade de Fortaleza, Ceará. Revista Brasileira de Epidemiologia 2004; 8(3):316-323.

14. Trelha CS, Nakaoski T, Franco SS, Dellaroza MSG, Yamada K, Cabresa M, Mesas AE, Gaetan CC. Capacidade funcional de idosos restritos ao domicílio, do conjunto Ruy Virmond Carnascialli, Londrina/PR. Seminário Ciências Biológicas e da Saúde, Londrina 2005; 26(1):37-46.

15. Reis S, Higino M. Condição de saúde bucal de idosos institucionalizados em Goiânia - GO, 2003. Rev Bras Epidemiol 2005; 8(1):67-73.

16. Slade GD, Locker D, Leake JL, Price AS, Chao I. Differences in oral health status between institutionalized and non-institutionalized older adults. Community Dent Oral Epidemiol 1990; 18(5):272276.

Artigo apresentado em 16/03/2011

Aprovado em 30/05/2011

Versão final apresentada em 08/06/2011 